\title{
INTEGRATED STUDY OF AD-NET MIE-LIDAR NETWORK AND DATA ASSIMILATED CTM FOR ASIAN DUST EPIDEMIOLOGY IN JAPAN
}

\author{
Atsushi Shimizu $^{1 *}$, Tomoaki Nishizawa ${ }^{l}$, Nobuo Sugimoto ${ }^{l}$, Ichiro Matsui ${ }^{l}$, \\ Hiroshi Kobayashi ${ }^{2}$, Takashi Maki ${ }^{3}$, Thomas T. Sekiyama ${ }^{3}$, Kumiko Kanatani ${ }^{4}$ \\ ${ }^{1}$ National Institute for Environmental Studies, 16-2 Onogawa, Tsukuba 305-8506 Japan \\ *Email:shimizua@nies.go.jp \\ ${ }^{2}$ University of Yamanashi, 4-4-37 Takeda, Kofu 400-8510 Japan \\ ${ }^{3}$ Meteorological Research Institute, 1-1 Nagamine, Tsukuba 305-0052 Japan \\ ${ }^{4}$ Kyoto University, Yoshida-konoe-cho, Sakyo-ku, Kyoto 606-8501 Japan
}

\begin{abstract}
Mie-scattering lidar data are going to be incorporated into data assimilation in chemical transport model to represent more reliable / useful horizontal distribution of Asian dust over Japan. The result is utilized in an epidemiology which surveys effect of Asian dust to human health. Wider application of AD-Net is expected in the field of environmental researches.
\end{abstract}

\section{INTRODUCTION}

Recent days Mie-scattering lidar data is utilized in Japan for environmental epidemiology on Asian dust [1]. Lidar is able to estimate concentrations of dust particles in fine time resolution using backscatter and depolarization ratio. It is compared with various medical dataset obtained in hospitals, or in domestic questionnaire based reports. Although several case studies were published, some limitations are recognized in these days. Especially, insufficient horizontal coverage is a problem in the epidemiology because patients are usually widely distributed. In this study, a data assimilation is introduced in a chemical transport model (CTM) with dust extinction coefficient by lidars, then the output on the grid points in CTM is supplied for comparison with dataset of health. Also, lidar system is updated to observe lower altitude to catch the actual air which is directly affect human beings. In addition, introduction of polarization optical particle counter (POPC) is planned. Finally, dust exposure estimated by lidar/POPC is distributed for public in real time.

\section{CURRENT STATUS OF AD-NET}

Dual wavelength polarization lidars are currently operated in 17 sites in East Asia. Each lidar measures backscatter at $532 / 1064 \mathrm{~nm}$ and depolarization ratio at $532 \mathrm{~m}$, with $30 \mathrm{~m}$ vertical resolution and 15 minutes time resolution. As operated automatically regardless of weather conditions, classification of scatterers including aerosols, clouds, rain drops, snow, and so on are practically important. For clear air region, Fernald's method is applied at $532 \mathrm{~nm}$ to retrieve aerosol backscatter (or extinction) coefficients and particulate depolarization ratio. Finally dust extinction and spherical particle extinction coefficients are estimated [2]. Results are uploaded to NIES WWW server, and distributed to the Ministry of Environment Japan every hour. Currently the lowest altitude of valid data is fixed at $120 \mathrm{~m}$ from surface.

\section{LIDAR DATA TREATMENT FOR HIND CAST EPIDEMIOLOGY}

Usually information related to human health are collected daily basis. Actual atmospheric condition changes in a day, thus a well-designed exposure data from atmospheric observation is needed. Lidar can measure atmosphere in high time resolution, but statistical approach is indispensable for epidemiology. Fig 1 is an example of time-height sections of backscatter coefficients and depolarization ratio accompanied with dust / spherical particle extinctions. These quantities are estimated in the time resolution of 15 minutes (96 times per day) in AD-Net, but just averaging over time in a day is not suitable because some kind of contamination from 
extremely large extinction by cloud / fog / snow are not excluded perfectly. Thus we introduced median value during one day in the initial stage of collaboration with epidemiology. However, this is not the best solution for drastically changing atmospheric conditions. For example, a heavy dust passed rapidly over lidar and clear air mass arrived just after dust, median cannot depict the short lived dust event at all. We have proposed two methods to conquer this difficulty.

(1) Averaging of segmented median

Median is obtained every 3 hours, then 8 values are averaged with weighting.

(2) Level counting by hourly basis

Hours in which dust extinction exceeds threshold value are counted in a day.

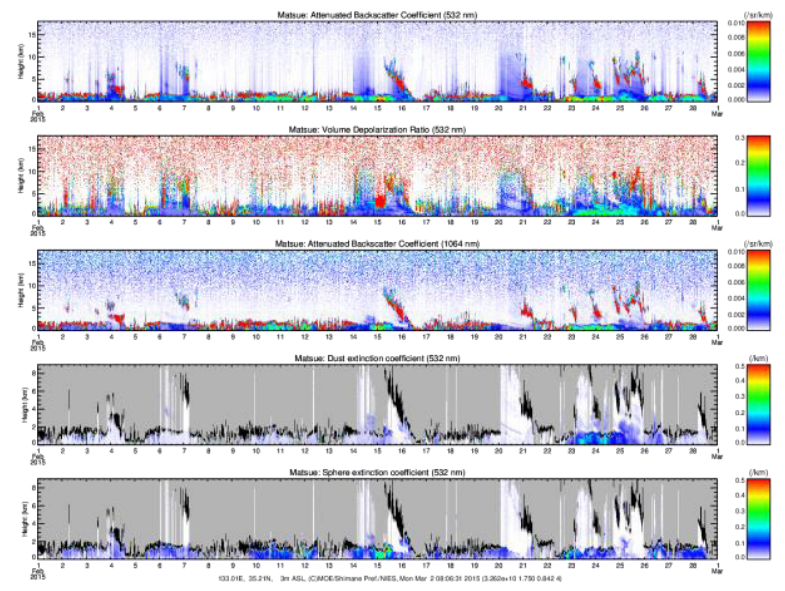

Figure 1. Time height indications of (from top) attenuated backscatter coefficient at 532 $\mathrm{nm}$, volume depolarization ratio at $532 \mathrm{~nm}$, attenuated backscatter coefficient at 1064nm, dust extinction coefficient, and spherical particle extinction coefficient observed in February 2015 at Matsue (133E, 35N).

\section{PLANS FOR OBTAINING MORE USEFUL DATA}

(1) Data assimilated CTM

Dust concentration can be calculated by chemical transport model (CTM) with appropriate meteorological data. If the horizontal grid scale of the model is small enough to represent the local exposure level in (for example) each city, the problem about distance between lidar location and colony of patients can be defeated. Then the accuracy of calculated concentration of dust is the key factor. Recently data assimilation technique is combined with CTM, and lidar data can be included in this system $[3,4]$. Thus, dust extinction estimated by the lidar network will raise the reliability of concentration predicted by CTM, and such results are the most suitable for epidemiology. We are now considering to make a long period assimilated dust concentration data in East Asian region.

(2) POPC

POPC measures size and depolarization ratio at 120 degree of independent particle at $780 \mathrm{~nm}$. As this equipment is operated at the surface, the results can be directly compared with information related to the human health. Challenging is the relationship with the number concentration by POPC and dust extinction by lidar or mass concentration (TSP etc.) of dust.

\section{(3) Smaller telescope for lidar}

Currently AD-Net does not estimate optical properties below $120 \mathrm{~m}$ altitude due to insufficient overlap of laser and FOV of the telescope. For epidemiology, information from lower altitude is more important because human breathes air at the surface. We additionally attach a smaller telescope with wider FOV for each lidar in order to retrieve optical properties around $60 \mathrm{~m}$ from the surface.

\section{CONCLUSIONS}

A plan to make lidar data more useful for an epidemiology related to Asia dust is explained. A closer application of lidar data in the civic life is demanded in Japan, and continuous improvements of equipment / data treatment are indespensable.

\section{ACKNOWLEDGEMENT}

This work is supported by the Environment Research and Technology Development Fund (51502) of the Ministry of Environment Japan.

\section{REFERENCES}

[1] Ueda, K., A. Shimizu, H. Nitta, and K. Inoue (2012), Long-range transported Asian Dust and emergency ambulance dispatches, Inhalation Toxicology, $24 \quad$ (12), 858-867, doi:10.3109/08958378.2012.724729. 
[2] Shimizu, A., N. Sugimoto, I. Matsui, K. Arao, I. Uno, T. Murayama, N. Kagawa, K. Aoki, A. Uchiyama, and A. Yamazaki (2004), Continuous observations of Asian dust and other aerosols by polarization lidars in China and Japan during ACE-Asia, J. Geophys. Res., 109 (D19), D19S17, doi:10.1029/2002JD003253.

[3] Yumimoto, K., I. Uno, N. Sugimoto, A. Shimizu, and S. Satake (2007), Adjoint inverse modeling of dust emission and transport over East Asia, Geophys. Res. Lett., 34, L08806, doi:10.1029/2006GL028551.

[4] Sekiyama, T. T., T. Y. Tanaka, A. Shimizu, and T. Miyoshi (2010), Data assimilation of CALIPSO aerosol observations, Atmos. Chem. Phys., 10 (1), 39-49, doi:10.5194/acp-10-39-2010. 